University of Nebraska - Lincoln

DigitalCommons@University of Nebraska - Lincoln

Ralph Skomski Publications

Research Papers in Physics and Astronomy

2010

Permanent magnetism of dense-packed nanostructures

Ralph Skomski

University of Nebraska-Lincoln, rskomski2@unl.edu

Yi Liu

University of Nebraska-Lincoln, yliu@unl.edu

Jeffrey E. Shield

University of Nebraska-Lincoln, jshield@unl.edu

George C. Hadjipanayis

University of Delaware, hadji@udel.edu

David J. Sellmyer

University of Nebraska-Lincoln, dsellmyer@unl.edu

Follow this and additional works at: https://digitalcommons.unl.edu/physicsskomski

Part of the Physics Commons

Skomski, Ralph; Liu, Yi; Shield, Jeffrey E.; Hadjipanayis, George C.; and Sellmyer, David J., "Permanent magnetism of dense-packed nanostructures" (2010). Ralph Skomski Publications. 63.

https://digitalcommons.unl.edu/physicsskomski/63

This Article is brought to you for free and open access by the Research Papers in Physics and Astronomy at DigitalCommons@University of Nebraska - Lincoln. It has been accepted for inclusion in Ralph Skomski Publications by an authorized administrator of DigitalCommons@University of Nebraska - Lincoln. 


\title{
Permanent magnetism of dense-packed nanostructures
}

\author{
R. Skomski, ${ }^{1, a)}$ Y. Liu, J. E. Shield, ${ }^{1}$ G. C. Hadjipanayis, ${ }^{2}$ and D. J. Sellmyer ${ }^{1}$ \\ ${ }^{1}$ Department of Physics and Astronomy and Nebraska Center for Materials and Nanoscience, University of \\ Nebraska, Lincoln, Nebraska 68588, USA \\ ${ }^{2}$ Department of Physics and Astronomy, University of Delaware, Newark, Delaware 19716, USA
}

(Presented 19 January 2010; received 31 October 2009; accepted 10 December 2009; published online 4 May 2010)

The effect of nanostructuring on magnetostatic interactions in permanent magnets is investigated by model calculations. Emphasis is on the energy product as a function of packing fraction of the magnetic phase, of the magnet's macroscopic shape, and of the nanoscale feature size. The main difference between nanostructured and macroscopic magnetic bodies, namely, the transition between coherent and incoherent reversal, has a far-reaching impact on demagnetizing field and energy product. For small magnet sizes, the energy product is substantially enlarged, up to $\mu_{0} M_{\mathrm{s}}{ }^{2} / 4$ for soft magnetic materials, but this effect is difficult to exploit in real devices. In bulk magnets, the energy product depends on the packing fraction $f$ of the soft phase and exhibits a maximum $\mu_{0} M_{\mathrm{s}}{ }^{2} / 12$ for $f=2 / 3$. Nanoscale magnetization processes involve demagnetizing factors different from the macroscopic ones used to determine the optimum shape of permanent magnets. Confusion of these two types of demagnetizing fields yields unphysical mechanisms, such as hysteresis-loop overskewing and the addition of self-interaction fields to the external field. (C) 2010 American Institute of Physics. [doi:10.1063/1.3337657]

\section{INTRODUCTION}

A key consideration in permanent magnetism is the volume fraction of the magnetic phase. This is because the energy product $(B H)_{\max }$ never exceeds $\mu_{0} M_{\mathrm{s}}{ }^{2} / 4$, where $M_{\mathrm{s}}$ is the volume-averaged saturation magnetization. The energy product determines the usable magnetostatic energy, stored outside the magnet, so that nonmagnetic regions, such as voids and nonmagnetic grain boundaries, must be included in the magnet volume. Most permanent-magnet materials of current interest are nanostructured, but typical devices are macroscopic and the energy product is realized by adjusting the magnet's shape and demagnetizing factor, ${ }^{1,2}$ which derives from Maxwell's equations. The packing fraction of the hard-magnetic phase and other structural features affect the magnetic field inside and outside the magnet, and question arises whether there is any interference between nanoscale and macroscopic features and how they could be used to improve the performance of permanent magnets. ${ }^{3-6}$ This also affects potential applications that use permanent magnets of reduced size, for example, in micromechanical devices and elements for spin electronics.

Typically, the energy product of a permanent magnet decreases with the volume fraction of the magnetic phase, which is a well-known challenge in the processing of permanent magnets. For example, the performance of polymerbonded permanent magnets is limited by the volume fraction of the magnetic particles, and sintered high-performance $\mathrm{Nd}-\mathrm{Fe}-\mathrm{B}$ magnets contain a Nd-rich grain-boundary phase that surrounds the grains but occupies a relatively small volume fraction. ${ }^{7,8}$ The first part of this paper deals with the less common case that nanoscale magnetostatic interactions

${ }^{\text {a)} E l e c t r o n i c ~ m a i l: ~ r s k o m s k i @ n e b . r r . c o m . ~}$ cause the energy product to increase with decreasing volume fraction. Since shape anisotropy is caused by magnetostatic self-interactions, high-magnetization materials may be used to create shape anisotropy. This effect is actually exploited in alnico magnets, which consist of FeCo needles in an AlNi matrix, ${ }^{9}$ and in fine-particle magnets ${ }^{10}$ produced by electrodeposition into a mercury cathode and intensively investigated in the mid-20th century. ${ }^{10}$ Element-strategic considerations addressing the limited availability of rare earths have lead to renewed interest in alternative materials, and it is interesting to explore the limitations of the magnetostatic approach.

\section{SIZE DEPENDENCE OF ENERGY PRODUCT}

Let us start with the dependence of the energy product on the size of the device or element. Maxwell's equations do not contain a nanomagnetic length scale, but the energy stored by a permanent magnet depends on the coercivity, which is a nanoscale phenomenon. If a fictitious motor had a size of only a few nanometers, then one could exploit the shape anisotropy of small particles and achieve energy products of the order of $1000 \mathrm{~kJ} / \mathrm{m}^{3}$ in soft magnets such as $\mathrm{Fe}$ and $\mathrm{FeCo}$. In more detail, ideal small particles have an energy product of $(B H)_{\max }=\frac{1}{4} \mu_{0} M_{\mathrm{s}}^{2}\left(1-9 D^{2}\right)$, meaning that energy product vanishes for spherical particles $(D=1 / 3)$. Long needles $(D=0)$ have a very impractical field distribution, but a prolate aspect ratio of $2: 1$ is sufficient to realize $73 \%$ of the maximum value $\frac{1}{4} \mu_{0} M_{\mathrm{s}}{ }^{2}$. Unfortunately, it is not possible to realize these energy products in macroscopic magnets, because shape anisotropy decreases as $A / R^{2}$ with increasing particle radius $R .^{8}$ Compaction of a powder consisting of very small particles would create magnetostatic fields that destroy the shape anisotropy. 


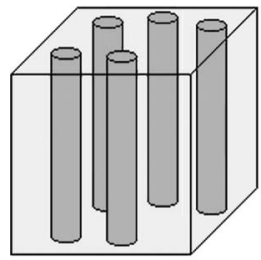

(a)

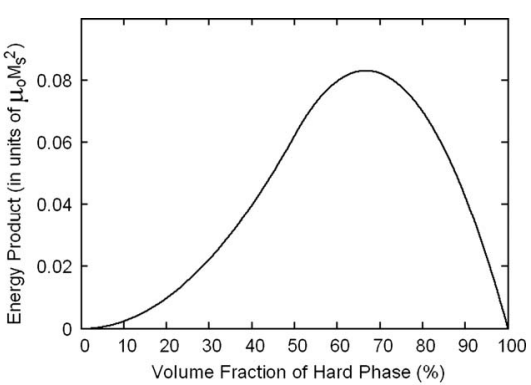

(b)
FIG. 1. Energy product of embedded wires: (a) alnico-type structure and (b) energy product as a function of packing fraction.

The compaction effect also limits the energy product of alnico permanent magnets, where FeCo needles of volume fraction $f$ are embedded in an AlNi matrix, as illustrated in Fig. 1(a). Assuming coherent rotation with rectangular hysteresis loops of coercivity $H_{\mathrm{c}}$, the energy product is equal to

$$
(B H)_{\max }=\frac{1}{4}\left(M_{\mathrm{r}} H_{\mathrm{c}}-H_{\mathrm{c}}^{2}\right)
$$

for $H_{\mathrm{c}}<M_{\mathrm{r}} / 2$ and

$$
(B H)_{\max }=\frac{1}{4} \mu_{0} M_{\mathrm{r}}^{2}
$$

for $H_{\mathrm{c}}>M_{\mathrm{r}} / 2$. In these equations, $M_{\mathrm{r}}=f M_{\mathrm{s}}$ is the remanence. In the Stoner-Wohlfarth limit, the coercivity of softmagnetic materials is equal to $\frac{1}{2}(1-3 D) M_{\mathrm{s}}$, and for a compact magnet of the type shown in Fig. 1(a), we can use, in fair approximation, $D=f / 3 .^{4,8}$ The corresponding coercivity, $H_{\mathrm{c}}=\frac{1}{2}(1-f) M_{\mathrm{s}}$, linearly interpolates between $M_{\mathrm{s}} / 2$ for $f=0$ (limit of isolated wires) and zero for $f=1$ (bulk soft magnet).

Substituting $H_{\mathrm{c}}=\frac{1}{2}(1-f) M_{\mathrm{s}}$ and $M_{\mathrm{r}}=f M_{\mathrm{s}}$ into Eq. (1) yields $(B H)_{\max }=\mu_{0} f^{2} M_{\mathrm{s}}^{2} / 4$ for $H_{\mathrm{c}}<M_{\mathrm{r}} / 2$ and $(B H)_{\max }$ $=\mu_{0}(1-f)(3 f-1) M_{\mathrm{s}}^{2} / 4$ for $H_{\mathrm{c}}>M_{\mathrm{r}} / 2$. Figure $1(\mathrm{~b})$ shows the energy product predicted by these equations. Very low packing factions lead to a waste of energy product due to an unnecessary dilution of the magnetization. For high volume fractions, the demagnetizing factor is close to that of a dense thin film, and the preferential magnetization direction turns into the film plane. The curve exhibits a maximum at the volume fraction of $f=2 / 3$, and the corresponding maximum energy product is

$$
(B H)_{\max }=\frac{1}{12} \mu_{0} M_{\mathrm{r}}^{2} \text {. }
$$

For $\mathrm{Fe}_{65} \mathrm{Co}_{35}$, this yields a value of $390 \mathrm{~kJ} / \mathrm{m}^{3}$ (49 MG Oe). However, this is an upper limit, similar to the difficult-toachieve value of more than $1000 \mathrm{~kJ} / \mathrm{m}^{3}$ for hard-soft nanostructures. ${ }^{11}$ Addition of uniaxial anisotropy with an easy axis parallel to the wires $\left(K_{1}>0\right)$ moves the peak toward higher volume fractions and higher energy-product values, whereas $K_{1}<0$ has the opposite effect.

\section{SIZE DEPENDENCE OF DEMAGNETIZING FIELD}

The model leading to Eq. (2) assumes that the magnetization reversal in the wires is coherent (Stoner-Wohlfarthtype). This is reasonable for thin wires, but when the wire radius exceeds the coherence radius $R_{\text {coh }}$, which is of the order of $10 \mathrm{~nm}$ for a broad range of materials, then the mechanism changes from coherent rotation to curling. ${ }^{8,12-15}$

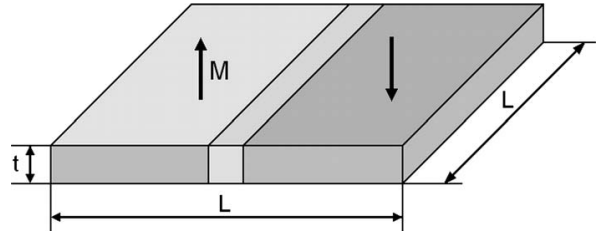

FIG. 2. Thin-film magnets where macroscopic demagnetizing fields cannot be defined because the micromagnetic length scales interfere with size of the magnet. If a demagnetizing factor is used to describe such magnets, one obtains unphysical predictions such as overskewing.

The curling reduces the nucleation field (coercivity) of a perfect $c$-axis-aligned uniaxial ellipsoid of revolution from

$$
H_{\mathrm{c}}=\frac{2 K_{1}}{\mu_{0} M_{\mathrm{s}}}+(1-3 D) M_{\mathrm{s}}
$$

to

$$
H_{\mathrm{c}}=\frac{2 K_{1}}{\mu_{0} M_{\mathrm{s}}}-D M_{\mathrm{s}}+\frac{c(D) A}{\mu_{0} M_{\mathrm{s}} R^{2}},
$$

where $A$ is the exchange stiffness and $c$ assumes values of 8.666 (spheres) and 6.6678 (long needles). ${ }^{8}$ These equations include the case of soft wires $\left(K_{1}=0\right)$. The curling mode is characterized by magnetic flux closure and therefore by a reduction in the magnetostatic self-energy. ${ }^{14}$ Compare, for example, Eqs. (3) and (4) for small and large spherical particles, respectively. Coherent rotation [Eq. (3)] then yields $H_{\mathrm{c}}=H_{\mathrm{a}}=2 K_{1} / \mu_{0} M_{\mathrm{s}}$, whereas curling [Eq. (4)] leads to $H_{\mathrm{c}}$ $=H_{\mathrm{a}}-M_{\mathrm{s}} / 3$. This means that the spherical demagnetizing field $-M_{\mathrm{s}} / 3$ is "missing" in the coherent-rotation limit.

The counterintuitive size dependence of the demagnetizing field can be traced to the demagnetizing field during coherent rotation. For example, the demagnetizing field in a small sphere is $-\mathbf{M} / 3$. Since the sphere's magnetostatic energy is independent of the magnetization direction, the demagnetization field does not enter any micromagnetic results. A more general argument is that $\mathbf{H}_{\mathrm{d}}=-\mathbf{M} / 3$ in $\mathbf{M} \cdot \mathbf{H}_{\mathrm{d}}$ yields an energy-density contribution proportional to $\mathbf{M}^{2}$ $=M_{\mathrm{s}}^{2}$, which is independent of the magnetization angles and amounts to a physically unimportant constant shift of the energy zero. In particular, it is not possible to consider selfinteraction fields as an addition to the external field.

\section{DISCUSSION AND CONCLUSIONS}

The term $-D M_{\mathrm{s}}$ in Eq. (4) is unrelated to the macroscopic demagnetizing factor but reflects the collapse of the magnetostatic self-interaction due to the curling-type flux closure. By contrast, the macroscopic demagnetizing factor describes the influence of the magnet's macroscopic poles on the magnetic field outside (and inside) the magnet. In this case, the argument based on $\mathbf{M}^{2}=M_{\mathrm{s}}{ }^{2}$ or $|\mathbf{M}|=M_{\mathrm{s}}$ no longer applies because the average magnetization is reduced by domain formation. A meaningful definition of a macroscopic demagnetizing field requires the magnetization to be reasonably homogeneous throughout the magnet, that is, the domains and domain walls must be much smaller than the macroscopic size of the magnet. Figure 2 shows a case where this requirement is not satisfied because the dimensions of the 


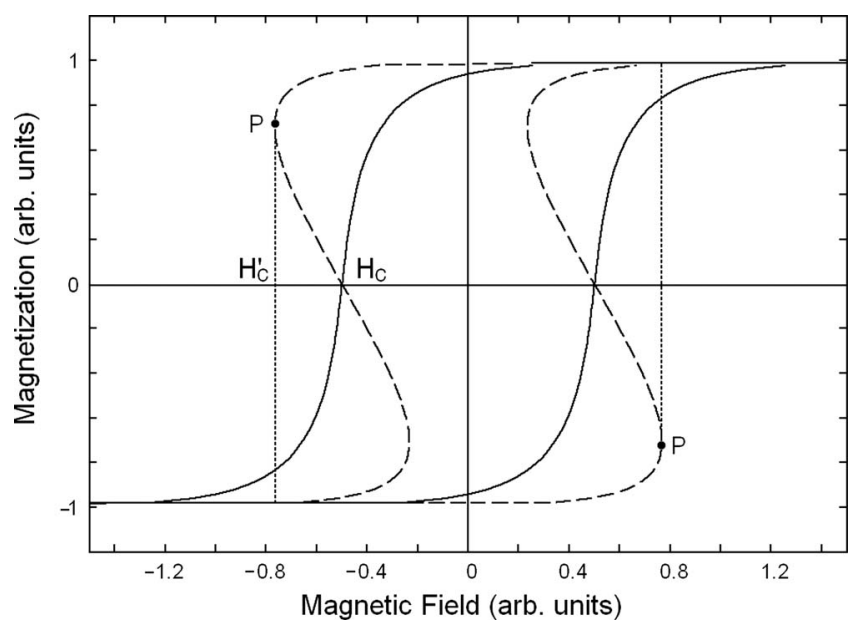

FIG. 3. Hysteresis-loop overskewing. The demagnetizing-field correction or "shearing" of a nearly rectangular uncorrected loop (solid line) yields an overskewed loop (dashed line) with instabilities $(P)$. In contrast to ordinary demagnetizing-field corrections, which leave the coercivity unchanged $\left(H_{\mathrm{c}}\right)$, the overskewing enhances the coercivity $\left(H_{\mathrm{c}}^{\prime}\right)$.

domains are comparable to the macroscopic size $L$ of the magnet. In a permanent magnet having the shape of an ellipsoid of revolution, the demagnetizing field is reasonably homogeneous. In a thin film, as in Fig. 2, the demagnetizing field is extremely inhomogeneous, and the use of a demagnetizing factor is a very poor approximation. The application of demagnetizing-field corrections may then give to illdefined predictions, such as overskewed hysteresis loops (Fig. 3). Overskewing tends to occur if uncorrected loops of films with perpendicular anisotropy are nearly rectangular. In practice, this amounts to a strongly reduced demagnetizingfield correction, but further research is necessary to specify this reduction for specific mechanism of magnetization reversal.

Recently, Dobrynin et $a l^{6}$ advocated the inclusion of magnetostatic self-interaction fields into micromagnetic simulations. This affects a number of seemingly wellunderstood magnetic properties, such as energy product and coercivity, and questions a broad range of past theoretical and experimental works. The unusual treatment of the selfenergy in Ref. 6 is motivated by the claim that magnetization processes in small particles are "discrete," that is, the magnetization changes directly from $-M_{\mathrm{s}}$ to $+M_{\mathrm{s}}$ or vice versa.
In fact, the Heisenberg exchange is sufficiently strong to ensure $\mathbf{M}^{2}=M_{\mathrm{s}}^{2}$ on an atomic scale, so that magnetization reversal is always realized by a rotation of the local magnetization. In other words, the assumption of discrete magnetization jumps crudely misinterprets the physics of magnetization reversal and corresponds to unphysical addition of the self-interaction field to the external field.

In conclusion, we have investigated how magnetostatic interactions affect energy product and demagnetizing fields in nanostructured permanent magnets. High energy products may be created in nanoscale devices, but compaction is generally a limiting consideration for soft-magnetic phases. In alnico-type magnets, a maximum energy product of $\mu_{0} M_{\mathrm{s}}{ }^{2} / 12$ is achieved for a volume fraction of $f=2 / 3$. The meaning of nanoscale demagnetizing factors qualitatively differs from that of the macroscopic demagnetizing factors usually considered in permanent magnetism. This is the reason for unphysical demagnetizing-field effects, such as overskewed hysteresis loops.

\section{ACKNOWLEDGMENTS}

Thanks are due to X.-Zh. Li for the discussion of some thin-film hysteresis loops. This research was supported by DOE (G.C.H. and D.J.S.), AFOSR (R.S. and J.E.S.), NSF MRSEC (R.S.), and NCMN (R.S.).

${ }^{1}$ J. A. Osborn, Phys. Rev. 67, 351 (1945).

${ }^{2}$ R. M. Bozorth, Ferromagnetism (van Nostrand, Princeton, NJ, 1951).

${ }^{3}$ R. Skomski, J.-P. Liu, and D. J. Sellmyer, Phys. Rev. B 60, 7359 (1999).

${ }^{4}$ R. Skomski, G. C. Hadjipanayis, and D. J. Sellmyer, IEEE Trans. Magn. 43, 2956 (2007).

${ }^{5}$ M. Gabay and G. C. Hadjipanayis, J. Appl. Phys. 101, 09K507 (2007).

${ }^{6}$ A. N. Dobrynin, V. M. T. S. Barthem, and D. Givord, Appl. Phys. Lett. 95, 052511 (2009)

${ }^{7}$ G. C. Hadjipanayis, in Rare-Earth-Iron Permanent Magnets, edited by J. M. D. Coey (Oxford University Press, Oxford, 1996), p. 286.

${ }^{8}$ R. Skomski and J. M. D. Coey, Permanent Magnetism (Institute of Physics, Bristol, 1999).

${ }^{9}$ R. A. McCurrie, Ferromagnetic Materials-Structure and Properties (Academic, London, 1994).

${ }^{10}$ W. H. Meiklejohn, Rev. Mod. Phys. 25, 302 (1953).

${ }^{11}$ R. Skomski and J. M. D. Coey, Phys. Rev. B 48, 15812 (1993).

${ }^{12}$ R. Skomski, J. Magn. Magn. Mater. 272-276, 1476 (2004).

${ }^{13}$ A. Aharoni, Rev. Mod. Phys. 34, 227 (1962).

${ }^{14} \mathrm{~A}$. Aharoni, Introduction to the Theory of Ferromagnetism (Oxford University Press, Oxford, 1996).

${ }^{15}$ R. Skomski, Simple Models of Magnetism (Oxford University Press, Oxford, 2008). 